УДК 517.9

\title{
On Uniqueness and Continuous Dependence on the Initial Data of the Solution of a System of Two Loaded Parabolic Equations with the Cauchy Data
}

\author{
Igor V. Frolenkov* \\ Irina S. Antipina ${ }^{\dagger}$ \\ Natalya M. Terskikh \\ Institute of Mathematics and Computer Science \\ Siberian Federal University \\ Svobodny, 79, Krasnoyarsk, 660041 \\ Russia
}

Received 17.01.2019, received in revised form 25.01.2019, accepted 03.02.2019

We study the Cauchy problem for the system of one-dimensional loaded parabolic equations. Uniqueness and continuous dependence of solutions on the initial data in the class of smooth bounded functions is proved.

Keywords: loaded equation, system of parabolic equation, weak approximation method, Cauchy problem, uniqueness, continuous dependence of the solution on the initial data.

DOI: 10.17516/1997-1397-2019-12-3-298-309.

\section{Introduction}

In [2] the sufficient conditions for solvability of the problem in the class of smooth bounded functions were obtained. A problem similar to the one-dimensional Burgers-type equation was considered in [3], where the question of the existence of solutions was also investigated.

In this paper we prove the uniqueness of the classical solution in the class of sufficiently smooth bounded functions and formulate the theorem of uniqueness. Also we study the continuous dependence of the solution on the right-hand side and the initial conditions and formulate the corresponding theorem.

\section{Statement of the problem}

In the space $E_{1}$ of variables $x$ choose $r$ different points $\alpha_{k}, k=\overline{1, r}$. In the strip $G_{[0, T]}=$ $=\left\{(t, x) \mid 0 \leqslant t \leqslant T, x \in E_{1}\right\}$ consider the Cauchy problem for the system of loaded non-classical parabolic equations

$$
\begin{gathered}
u_{t}(t, x)=a_{1}(t) u_{x x}(t, x)+b_{1}(t) u_{x}(t, x)+f_{1}\left(t, x, u, v, \bar{\varphi}_{u}(t), \bar{\varphi}_{v}(t)\right), \\
v_{t}(t, x)=a_{2}(t) v_{x x}(t, x)+b_{2}(t) v_{x}(t, x)+f_{2}\left(t, x, u, v, \bar{\varphi}_{u}(t), \bar{\varphi}_{v}(t)\right),
\end{gathered}
$$

\footnotetext{
*igor@frolenkov.ru

†irina.antipina.21@mail.ru

$\ddagger$ nt.terskikh@mail.ru

(C) Siberian Federal University. All rights reserved
} 


$$
u(0, x)=u_{0}(x), \quad v(0, x)=v_{0}(x), \quad x \in E_{1},
$$

here the components of vector-functions

$$
\bar{\varphi}_{u}(t)=\left(u\left(t, \alpha_{k}\right), \frac{\partial^{j}}{\partial x^{j}} u\left(t, \alpha_{k}\right)\right), \quad \bar{\varphi}_{v}(t)=\left(v\left(t, \alpha_{k}\right), \frac{\partial^{j}}{\partial x^{j}} v\left(t, \alpha_{k}\right)\right), \quad k=\overline{1, r}, j=\overline{0, p_{1}},
$$

are traces of functions $u(t, x), v(t, x)$ and all their derivatives with respect to $x$ up to order $p_{1}$.

Denote by $Z_{x}^{p}\left(G_{[0, T]}\right)$ the set of the functions $u(t, x)$ defined in $G_{[0, T]}$ belonging to the class

$$
C_{t, x}^{1, p}=\left\{u(t, x) \mid \frac{\partial u}{\partial t}, \frac{\partial^{j} u}{\partial x^{j}} \in C\left(G_{[0, T]}\right), j=0, \ldots, p\right\},
$$

and bounded in $(t, x) \in G_{[0, T]}$ together with their derivatives,

$$
\sum_{j=0}^{p}\left|\frac{\partial^{j} u(t, x)}{\partial x^{j}}\right| \leqslant C
$$

\section{Uniqueness of solution}

In [4] the sufficient conditions for the existence of solutions of the problem (1) and (2) in the class $Z_{x}^{p}\left(G_{\left[0, t^{*}\right]}\right)$ are obtained. Here $0<t^{*} \leqslant T$ is a fixed constant. Suppose that $p \geqslant$ $\max \left\{p_{1}, 2\right\}++2 \geqslant 4$, and with this $p$ satisfies the conditions of the existence theorem [4]. In view of this theorem a classical solution $u^{1}(t, x) \in Z_{x}^{p}\left(G_{\left[0, t^{*}\right]}\right), v^{1}(t, x) \in Z_{x}^{p}\left(G_{\left[0, t^{*}\right]}\right)$ of the problem $(1),(2)$ exists. We prove that this solution is unique. Suppose that there is a pair of functions $u^{2}(t, x) \in Z_{x}^{p}\left(G_{\left[0, t^{*}\right]}\right), v^{2}(t, x) \in Z_{x}^{p}\left(G_{\left[0, t^{*}\right]}\right)$, along with the functions $u^{1}(t, x), v^{1}(t, x)$, which is a solution of the system of equations. Then

$$
\begin{gathered}
u_{t}^{i}(t, x)=a_{1}(t) u_{x x}^{i}(t, x)+b_{1}(t) u_{x}^{i}(t, x)+f_{1}\left(t, x, u^{i}, v^{i}, \bar{\varphi}_{u^{i}}(t), \bar{\varphi}_{v^{i}}(t)\right), \\
v_{t}^{i}(t, x)=a_{2}(t) v_{x x}^{i}(t, x)+b_{2}(t) v_{x}^{i}(t, x)+f_{2}\left(t, x, u^{i}, v^{i}, \bar{\varphi}_{u^{i}}(t), \bar{\varphi}_{v^{i}}(t)\right), \\
u^{i}(0, x)=u_{0}^{i}(x), \quad v^{i}(0, x)=v_{0}^{i}(x), \quad x \in E_{1}, i=1,2 .
\end{gathered}
$$

Suppose that the functions $a_{1}(t), a_{2}(t), b_{1}(t), b_{2}(t), u_{0}(x), v_{0}(x)$, are real-valued, defined in $[0, T], G_{[0, T]}, E_{1}$, respectively and have all continuous derivatives occurring in the following relation and satisfying it

$$
\left|a_{1}(t)\right|+\left|a_{2}(t)\right|+\left|b_{1}(t)\right|+\left|b_{2}(t)\right|+\sum_{k=0}^{p+2}\left|\frac{d^{k}}{d x^{k}} u_{0}(x)\right|+\sum_{k=0}^{p+2}\left|\frac{d^{k}}{d x^{k}} v_{0}(x)\right| \leqslant C .
$$

Functions $f_{1}$ and $f_{2}$ are real-valued, defined and continuous for any values of their arguments. For all $t_{1} \in[0, T], u(t, x), v(t, x) \in Z^{p+2}\left(\left[0, t_{1}\right]\right)$ these functions as functions of the variables $(t, x) \in G_{\left[0, t_{1}\right]}$ are continuous and have continuous derivatives occurring in the relation

$$
\sum_{j=0}^{p+2}\left(\left|\frac{d^{j}}{d x^{j}} f_{1}\left(t, x, u, v, \bar{\varphi}_{u}(t), \bar{\varphi}_{v}(t)\right)\right|+\left|\frac{d^{j}}{d x^{j}} f_{2}\left(t, x, u, v, \bar{\varphi}_{u}(t), \bar{\varphi}_{v}(t)\right)\right|\right) \leqslant C(u, v),
$$

where the constant $C(u, v)$ depends on the functions $u(t, x), v(t, x)$. 
The difference $u^{1}(t, x)-u^{2}(t, x)=U(t, x), v^{1}(t, x)-v^{2}(t, x)=V(t, x)$ is a solution of the system of equations

$$
\begin{aligned}
& U_{t}(t, x)= a_{1}(t) U_{x x}(t, x)+b_{1}(t) U_{x}(t, x)+ \\
&+f_{1}\left(t, x, u^{1}, v^{1}, \bar{\varphi}_{u^{1}}(t), \bar{\varphi}_{v^{1}}(t)\right)-f_{1}\left(t, x, u^{2}, v^{2}, \bar{\varphi}_{u^{2}}(t), \bar{\varphi}_{v^{2}}(t)\right), \\
& V_{t}(t, x)= a_{2}(t) V_{x x}(t, x)+b_{2}(t) V_{x}(t, x)+ \\
&+f_{2}\left(t, x, u^{1}, v^{1}, \bar{\varphi}_{u^{1}}(t), \bar{\varphi}_{v^{1}}(t)\right)-f_{2}\left(t, x, u^{2}, v^{2}, \bar{\varphi}_{u^{2}}(t), \bar{\varphi}_{v^{2}}(t)\right), \\
& U(0, x)=0, \quad V(0, x)=0, \quad x \in E_{1} .
\end{aligned}
$$

Condition 1. Suppose that the function $f_{1}, f_{2}$ such that $\forall t_{1} \in(0, T], \forall u^{1}(t, x), u^{2}(t, x) \in$ $Z_{x}^{p}\left(G_{\left[0, t_{1}\right]}\right), \forall v^{1}(t, x), v^{2}(t, x) \in Z_{x}^{p}\left(G_{\left[0, t_{1}\right]}\right)$, the following relations hold:

$$
\begin{gathered}
f_{1}\left(t, x, u^{1}, v^{1}, \bar{\varphi}_{u^{1}}(t), \bar{\varphi}_{v^{1}}(t)\right)-f_{1}\left(t, x, u^{2}, v^{2}, \bar{\varphi}_{u^{2}}(t), \bar{\varphi}_{v^{2}}(t)\right)= \\
=\left(u^{1}-u^{2}\right) \cdot F^{1}+\sum_{k=1}^{r} \sum_{s=0}^{p_{1}}\left(\frac{\partial^{s}}{\partial x^{s}} u^{1}\left(t, \alpha_{k}\right)-\frac{\partial^{s}}{\partial x^{s}} u^{2}\left(t, \alpha_{k}\right)\right) \cdot F_{k, s}^{1}+ \\
+\left(v^{1}-v^{2}\right) \cdot G^{1}+\sum_{k=1}^{r} \sum_{s=0}^{p_{1}}\left(\frac{\partial^{s}}{\partial x^{s}} v^{1}\left(t, \alpha_{k}\right)-\frac{\partial^{s}}{\partial x^{s}} v^{2}\left(t, \alpha_{k}\right)\right) \cdot G_{k, s}^{1}, \\
f_{2}\left(t, x, u^{1}, v^{1}, \bar{\varphi}_{u^{1}}(t), \bar{\varphi}_{v^{1}}(t)\right)-f_{2}\left(t, x, u^{2}, v^{2}, \bar{\varphi}_{u^{2}}(t), \bar{\varphi}_{v^{2}}(t)\right)= \\
=\left(u^{1}-u^{2}\right) \cdot F^{2}+\sum_{k=1}^{r} \sum_{s=0}^{p_{1}}\left(\frac{\partial^{s}}{\partial x^{s}} u^{1}\left(t, \alpha_{k}\right)-\frac{\partial^{s}}{\partial x^{s}} u^{2}\left(t, \alpha_{k}\right)\right) \cdot F_{k, s}^{2}+ \\
+\left(v^{1}-v^{2}\right) \cdot G^{2}+\sum_{k=1}^{r} \sum_{s=0}^{p_{1}}\left(\frac{\partial^{s}}{\partial x^{s}} v^{1}\left(t, \alpha_{k}\right)-\frac{\partial^{s}}{\partial x^{s}} v^{2}\left(t, \alpha_{k}\right)\right) \cdot G_{k, s}^{2} .
\end{gathered}
$$

Condition 2. The functions $F^{1}, F_{k, s}^{1}, G^{1}, G_{k, s}^{1}, F^{2}, F_{k, s}^{2}, G^{2}, G_{k, s}^{2}$, where $k=\overline{1, r}, s=\overline{0, p_{1}}$ are known and sufficiently smooth, depend on $t, x, u^{1}(t, x), u^{2}(t, x), v^{1}(t, x), v^{2}(t, x), \bar{\varphi}_{u^{1}}(t)$, $\bar{\varphi}_{u^{2}}(t), \bar{\varphi}_{v^{1}}(t), \bar{\varphi}_{v^{2}}(t)$ and have all continuous derivatives occurring in the following relation and satisfying it:

$$
\begin{aligned}
& \sum_{j=0}^{p_{1}}\left(\left|\frac{\partial^{j}}{\partial x^{j}} F^{1}\right|+\left|\frac{\partial^{j}}{\partial x^{j}} G^{1}\right|+\left|\frac{\partial^{j}}{\partial x^{j}} F^{2}\right|+\left|\frac{\partial^{j}}{\partial x^{j}} G^{2}\right|+\right. \\
& \left.+\sum_{k=1}^{r} \sum_{s=0}^{p_{1}}\left(\left|\frac{\partial^{j}}{\partial x^{j}} F_{k, s}^{1}\right|+\left|\frac{\partial^{j}}{\partial x^{j}} G_{k, s}^{1}\right|+\left|\frac{\partial^{j}}{\partial x^{j}} F_{k, s}^{2}\right|+\left|\frac{\partial^{j}}{\partial x^{j}} G_{k, s}^{2}\right|\right)\right) \leqslant C, \quad \forall(t, x) \in G_{\left[0, t_{1}\right]} .
\end{aligned}
$$

$C$ is a constant independent of $U(t, x), V(t, x)$ and their derivatives.

Theorem of uniqueness of the solution. If a solution of the system of equations (1) and (2) exists in the class $Z_{x}^{p}\left(G_{\left[0, t^{*}\right]}\right)$, where $p \geqslant \max \left\{p_{1}, 2\right\}+2 \geqslant 4$, then under conditions 1, 2 it is unique in the class $Z_{x}^{p}\left(G_{\left[0, t^{*}\right]}\right)$.

Proof. Represent the system of equations (4) in the following form

$$
\begin{aligned}
& U_{t}(t, x)=a_{1}(t) U_{x x}(t, x)+b_{1}(t) U_{x}(t, x)+U(t, x) F^{1}+ \\
& +\sum_{k=1}^{r} \sum_{s=0}^{p_{1}}\left(\frac{\partial^{s}}{\partial x^{s}} U\left(t, a_{k}\right) F_{k, s}^{1}\right)+V(t, x) G^{1}+\sum_{k=1}^{r} \sum_{s=0}^{p_{1}}\left(\frac{\partial^{s}}{\partial x^{s}} V\left(t, a_{k}\right) G_{k, s}^{1}\right),
\end{aligned}
$$




$$
\begin{aligned}
& V_{t}(t, x)=a_{1}(t) V_{x x}(t, x)+b_{1}(t) V_{x}(t, x)+U(t, x) F^{2}+ \\
& \quad+\sum_{k=1}^{r} \sum_{s=0}^{p_{1}}\left(\frac{\partial^{s}}{\partial x^{s}} U\left(t, a_{k}\right) F_{k, s}^{2}\right)+V(t, x) G^{2}+\sum_{k=1}^{r} \sum_{s=0}^{p_{1}}\left(\frac{\partial^{s}}{\partial x^{s}} V\left(t, a_{k}\right) G_{k, s}^{2}\right) .
\end{aligned}
$$

Introduce the non-negative, non-decreasing on $\left[0, t^{*}\right]$ functions

$$
m_{s}^{1}(t)=\sup _{G_{[0, t]}}\left|\frac{\partial^{s}}{\partial x^{s}} U(\xi, x)\right|, \quad m_{s}^{2}(t)=\sup _{G_{[0, t]}}\left|\frac{\partial^{s}}{\partial x^{s}} V(\xi, x)\right|, s=0,1, \ldots, p_{1} .
$$

By the maximum principle, for $(\xi, x) \in G_{\left[0, t^{*}\right]}, 0 \leqslant t \leqslant t^{*}$ we obtain

$$
\begin{aligned}
|U(\xi, x)| \leqslant \exp \left(\xi \cdot \sup _{\left.G_{\left[0, t^{*}\right]}\left|F^{1}\right|\right)}\right. & \left(\sum_{k=1}^{r} \sum_{s=0}^{p_{1}} \sup _{G_{\left[0, t^{*}\right]}}\left|F_{k, s}^{1}\right| \cdot m_{s}^{1}(t)+\right. \\
& \left.+m_{0}^{2}(t) \cdot \sup _{G_{\left[0, t^{*}\right]}}\left|G^{1}\right|+\sum_{k=1}^{r} \sum_{s=0}^{p_{1}} \sup _{G_{\left[0, t^{*}\right]}}\left|G_{k, s}^{1}\right| \cdot m_{s}^{2}(t)\right) \cdot \xi \\
|V(\xi, x)| \leqslant \exp \left(\xi \cdot \sup _{G_{\left[0, t^{*}\right]}}\left|G^{2}\right|\right) & \cdot\left(\sum_{k=1}^{r} \sum_{s=0}^{p_{1}} \sup _{G_{\left[0, t^{*}\right]}}\left|G_{k, s}^{2}\right| \cdot m_{s}^{2}(t)+\right. \\
& \left.+m_{0}^{1}(t) \cdot \sup _{G_{\left[0, t^{*}\right]}}\left|F^{2}\right|+\sum_{k=1}^{r} \sum_{s=0}^{p_{1}} \sup _{G_{\left[0, t^{*}\right]}}\left|F_{k, s}^{2}\right| \cdot m_{s}^{2}(t)\right) \cdot \xi .
\end{aligned}
$$

For $(\xi, x) \in G_{\left[0, t^{*}\right]}, 0 \leqslant t \leqslant t^{*}$ we have

$$
|U(\xi, x)| \leqslant C_{1} \cdot\left(\sum_{s=0}^{p_{1}} m_{s}^{1}(t)+\sum_{s=0}^{p_{1}} m_{s}^{2}(t)\right) \cdot t,|V(\xi, x)| \leqslant C_{2} \cdot\left(\sum_{s=0}^{p_{1}} m_{s}^{2}(t)+\sum_{s=0}^{p_{1}} m_{s}^{1}(t)\right) \cdot t .
$$

Apply $\sup _{G_{[0, t]}}$ to the both sides of these inequalities, due to the fact that the functions $m_{s}^{1}(t)$, $m_{s}^{2}(t), s=0,1, \ldots, p_{1}, 0 \leqslant t \leqslant t^{*}$ are nonnegative, we obtain:

$$
m_{0}^{1}(t) \leqslant C_{1} \cdot\left(\sum_{s=0}^{p_{1}} m_{s}^{1}(t)+\sum_{s=0}^{p_{1}} m_{s}^{2}(t)\right) \cdot t, m_{0}^{2}(t) \leqslant C_{2} \cdot\left(\sum_{s=0}^{p_{1}} m_{s}^{2}(t)+\sum_{s=0}^{p_{1}} m_{s}^{1}(t)\right) \cdot t .
$$

We differentiate the equations $j$ times wrt $x, j=1, \ldots, p_{1}$

$$
\begin{aligned}
\frac{\partial^{j}}{\partial x^{j}} U_{t}(t, x)= & a_{1} \frac{\partial^{j+2}}{\partial x^{j+2}} U(t, x)+b_{1} \frac{\partial^{j+1}}{\partial x^{j+1}} U(t, x)+ \\
& +\sum_{l=0}^{j}\left(C_{j}^{l} \frac{\partial^{l}}{\partial x^{l}} U(t, x) \frac{\partial^{j-l}}{\partial x^{j-l}} F^{1}\right)+\sum_{k=1}^{n} \sum_{s=0}^{p_{1}} \frac{\partial^{s}}{\partial x^{s}} U\left(t, \alpha_{k}\right) \frac{\partial^{j}}{\partial x^{j}} F_{k, s}^{1}+ \\
& +\sum_{l=0}^{j}\left(C_{j}^{l} \frac{\partial^{l}}{\partial x^{l}} V(t, x) \frac{\partial^{j-l}}{\partial x^{j-l}} G^{1}\right)+\sum_{k=1}^{n} \sum_{s=0}^{p_{1}} \frac{\partial^{s}}{\partial x^{s}} V\left(t, \alpha_{k}\right) \frac{\partial^{j}}{\partial x^{j}} G_{k, s}^{1}, \\
\frac{\partial^{j}}{\partial x^{j}} V_{t}(t, x)= & a_{2} \frac{\partial^{j+2}}{\partial x^{j+2}} V(t, x)+b_{2} \frac{\partial^{j+1}}{\partial x^{j+1}} V(t, x)+ \\
& +\sum_{l=0}^{j}\left(C_{j}^{l} \frac{\partial^{l}}{\partial x^{l}} U(t, x) \frac{\partial^{j-l}}{\partial x^{j-l}} F^{2}\right)+\sum_{k=1}^{n} \sum_{s=0}^{p_{1}} \frac{\partial^{s}}{\partial x^{s}} U\left(t, \alpha_{k}\right) \frac{\partial^{j}}{\partial x^{j}} F_{k, s}^{2}+ \\
& +\sum_{l=0}^{j}\left(C_{j}^{l} \frac{\partial^{l}}{\partial x^{l}} V(t, x) \frac{\partial^{j-l}}{\partial x^{j-l}} G^{2}\right)+\sum_{k=1}^{n} \sum_{s=0}^{p_{1}} \frac{\partial^{s}}{\partial x^{s}} V\left(t, \alpha_{k}\right) \frac{\partial^{j}}{\partial x^{j}} G_{k, s}^{2} .
\end{aligned}
$$


In view of the maximum principle, we obtain the similar estimates:

$$
\begin{aligned}
& m_{j}^{1}(t) \leqslant C_{1} \cdot\left(\sum_{s=0}^{p_{1}} m_{s}^{1}(t)+\sum_{s=0}^{p_{1}} m_{s}^{2}(t)\right) \cdot t, \\
& m_{j}^{2}(t) \leqslant C_{2} \cdot\left(\sum_{s=0}^{p_{1}} m_{s}^{1}(t)+\sum_{s=0}^{p_{1}} m_{s}^{2}(t)\right) \cdot t,
\end{aligned}
$$

Then we add these inequalities and get

$$
\sum_{s=0}^{p_{1}}\left(m_{s}^{1}(t)+m_{s}^{2}(t)\right) \leqslant \bar{C} \cdot\left(\sum_{s=0}^{p_{1}}\left(m_{s}^{1}(t)+m_{s}^{2}(t)\right)\right) \cdot t, \quad s=1, \ldots, p_{1} .
$$

Hence for $t \in[0, \theta]$, where $\theta<\frac{1}{C}$, it follows that

$$
\sum_{s=0}^{p_{1}}\left(m_{s}^{1}(t)+m_{s}^{2}(t)\right)=0 .
$$

As $m_{s}^{1}(t) \geqslant 0, m_{s}^{2}(t) \geqslant 0$, then for all $(t, x) \in G_{[0, \theta]}$ we have

$$
U(t, x)=0, \quad V(t, x)=0 .
$$

Similarly, because $\bar{C}$ does not depend on $\theta$ for $t \in[\theta, 2 \theta]$ we obtain that

$$
U(t, x)=0, \quad V(t, x)=0, \quad(t, x) \in G_{[0,2 \theta]} .
$$

After the finite number of steps, we obtain

$$
U(t, x) \equiv 0, \quad V(t, x) \equiv 0, \quad(t, x) \in G_{\left[0, t^{*}\right]} .
$$

Hence it follows that

$$
u^{1}(t, x)=u^{2}(t, x), \quad v^{1}(t, x)=v^{2}(t, x), \quad t \in\left[0, t^{*}\right], \quad x \in E_{1} .
$$

Therefore, the solutions coincide in the whole domain $G_{\left[0, t^{*}\right]}$ in the class $Z_{x}^{p}\left(G_{\left[0, t^{*}\right]}\right)$. The uniqueness theorem is proved.

Consider the example of an inverse problem for a system of parabolic equations for which the existence of the solution was investigated in [5].

Example. In the strip $\Pi_{[0, T]}=\left\{(t, x) \mid t \in[0, T], x \in E_{1}\right\}$ we consider the problem of finding real-valued functions $U(t, x), V(t, x), g_{i}(t), i=1,2$, satisfying the system of equations

$$
\begin{aligned}
U_{t} & =U_{x x}+b_{11}(t) U^{2}+b_{12}(t) V+g_{1}(t) m_{1}(t, x), \\
V_{t} & =V_{x x}+b_{21}(t) U+b_{22}(t) V^{2}+g_{2}(t) m_{2}(t, x),
\end{aligned}
$$

the initial conditions

$$
U(0, x)=U_{0}(x), \quad V(0, x)=V_{0}(x), \quad x \in E_{1} .
$$

The solution satisfies the overdetermination condition

$$
U(t, 0)=\beta_{1}(t), \quad V(t, 0)=\beta_{2}(t), \quad t \in[0, T],
$$


where $b_{i j}(t), m_{i}(t, x), U_{0}(x), V_{0}(x), \beta_{i}(t), i, j=1,2$ are given real-valued functions. Let the consistency conditions be fulfilled

$$
U_{0}(0)=\beta_{1}(0), \quad V_{0}(0)=\beta_{2}(0) .
$$

Let the following condition hold

$$
\left|m_{i}(t, 0)\right| \geqslant \delta>0, \quad i=1,2, \quad t \in[0, T], \quad \delta \text { - const. }
$$

All input data are real-valued, sufficiently smooth and bounded functions with their derivatives in $\Pi_{[0, T]}$.

The problem (12), (13) is reduced to the auxiliary direct problem

$$
\begin{aligned}
& U_{t}=U_{x x}+b_{11}(t) U^{2}+b_{12}(t) V+ \\
& +m_{1}(t, x) m_{1}^{-1}(t, 0)\left(\beta_{1}^{\prime}(t)-U_{x x}(t, 0)-b_{11}(t) \beta_{1}^{2}(t)-b_{12}(t) V(t, 0)\right), \\
& \begin{aligned}
V_{t}=V_{x x}+b_{21}(t) U & +b_{22}(t) V^{2}+ \\
& +m_{2}(t, x) m_{2}^{-1}(t, 0)\left(\beta_{2}^{\prime}(t)-V_{x x}(t, 0)-b_{21}(t) U(t, 0)-b_{22}(t) \beta_{2}^{2}(t)\right), \\
& U(0, x)=U_{0}(x), \quad V(0, x)=V_{0}(x) .
\end{aligned}
\end{aligned}
$$

Differences $W(t, x)=U_{1}(t, x)-U_{2}(t, x), Q(t, x)=V_{1}(t, x)-V_{2}(t, x)$ are a solution of the problem

$$
\begin{gathered}
W_{t}=W_{x x}+b_{11}(t) W\left(U^{1}+U^{2}\right)+b_{12}(t) Q+m_{1}(t, x) m_{1}^{-1}(t, 0)\left(-W_{x x}(t, 0)-b_{12}(t) Q(t, 0)\right), \\
Q_{t}=Q_{x x}+b_{21}(t) W+b_{22}(t) Q\left(V^{1}+V^{2}\right)+m_{2}(t, x) m_{2}^{-1}(t, 0)\left(-Q_{x x}(t, 0)-b_{21}(t) W(t, 0)\right), \\
W(0, x)=0, \quad Q(0, x)=0 .
\end{gathered}
$$

Check the conditions of the Theorem

$$
\begin{aligned}
& f_{1}\left(t, x, U^{1}, V^{1}, \bar{\varphi}_{U^{1}}(t), \bar{\varphi}_{V^{1}}(t)\right)-f_{1}\left(t, x, U^{2}, V^{2}, \bar{\varphi}_{U^{2}}(t), \bar{\varphi}_{V^{2}}(t)\right)= \\
& \quad=\left(U^{1}-U^{2}\right) F^{1}+\left(U_{x x}^{1}(t, 0)-U_{x x}^{2}(t, 0)\right) F_{1,2}^{1}+\left(V^{1}-V^{2}\right) G^{1}+\left(V^{1}(t, 0)+V^{2}(t, 0)\right) G_{1,0}^{1}, \\
& f_{2}\left(t, x, U^{1}, V^{1}, \bar{\varphi}_{U^{1}}(t), \bar{\varphi}_{V^{1}}(t)\right)-f_{2}\left(t, x, U^{2}, V^{2}, \bar{\varphi}_{U^{2}}(t), \bar{\varphi}_{V^{2}}(t)\right)= \\
& \quad=\left(U^{1}-U^{2}\right) F^{2}+\left(U^{1}(t, 0)+U^{2}(t, 0)\right) F_{1,0}^{2}+\left(V^{1}-V^{2}\right) G^{2}+\left(V_{x x}^{1}(t, 0)-V_{x x}^{2}(t, 0)\right) G_{1,2}^{2},
\end{aligned}
$$

where

$$
\begin{gathered}
F^{1}=b_{11}(t)\left(U^{1}+U^{2}\right), F_{1,2}^{1}=-m_{1}(t, x) m_{1}^{-1}(t, 0), F^{2}=b_{21}, F_{1,0}^{2}=-b_{21} m_{2}(t, x) m_{2}^{-1}(t, 0), \\
G^{1}=b_{12}, G^{2}=b_{22}(t)\left(V^{1}+V^{2}\right), G_{1,0}^{1}=-b_{12} m_{1}(t, x) m_{1}^{-1}(t, 0), G_{1,2}^{2}=-m_{2}(t, x) m_{2}^{-1}(t, 0),
\end{gathered}
$$

are known, sufficiently smooth and bounded functions.

Conditions 1, 2 of the Theorem of uniqueness of the solution are fulfilled. Hence, the solution of the problem (12), (13) is unique.

\section{Continuous dependence of the solution on the initial data}

In the space $E_{1}$ of variables $x$ choose $r$ different points $\alpha_{k}, k=\overline{1, r}$. Consider the Cauchy problem for the system of loaded non-classical parabolic equations 


$$
\begin{gathered}
U_{t}(t, x)=a_{1}(t) U_{x x}(t, x)+b_{1}(t) U_{x}(t, x)+U(t, x) F^{1}(t, x)+F^{3}(t, x)+V(t, x) G^{1}(t, x)+ \\
+\sum_{k=1}^{r} \sum_{s=0}^{p_{1}} \frac{\partial^{s}}{\partial x^{s}} U\left(t, a_{k}\right) F_{k, s}^{1}(t, x)+\sum_{k=1}^{r} \sum_{s=0}^{p_{1}} \frac{\partial^{s}}{\partial x^{s}} V\left(t, a_{k}\right) G_{k, s}^{1}(t, x), \\
V_{t}(t, x)=a_{2}(t) V_{x x}(t, x)+b_{2}(t) V_{x}(t, x)+U(t, x) F^{2}(t, x)+G^{3}(t, x)+V(t, x) G^{2}(t, x)+ \\
+\sum_{k=1}^{r} \sum_{s=0}^{p_{1}} \frac{\partial^{s}}{\partial x^{s}} U\left(t, a_{k}\right) F_{k, s}^{2}(t, x)+\sum_{k=1}^{r} \sum_{s=0}^{p_{1}} \frac{\partial^{s}}{\partial x^{s}} V\left(t, a_{k}\right) G_{k, s}^{2}(t, x), \\
U(0, x)=U_{0}(x), V(0, x)=V_{0}(x), x \in E_{1} .
\end{gathered}
$$

We choose and fix $p \geqslant \max \left\{p_{1}, 2\right\} \geqslant 2$. Suppose that the functions $a_{i}(t), b_{i}(t), U_{0}(x), V_{0}(x)$, $i, j=1,2$ are real-valued, defined in $[0, T], G_{[0, T]}, E_{1}$, respectively and have all continuous derivatives occurring in the following relation and satisfying it

$$
\left|a_{i}(t)\right|+\left|b_{i}(t)\right|+\sum_{k=0}^{p+2}\left|\frac{d^{k}}{d x^{k}} U_{0}(x)\right|+\sum_{k=0}^{p+2}\left|\frac{d^{k}}{d x^{k}} V_{0}(x)\right| \leqslant C .
$$

Constants $C$ are different and independent of the splitting parameter $\tau, U_{0}(x), V_{0}(x), F^{3}(t, x)$, $G^{3}(t, x), U(t, x), V(t, x)$ and their derivatives here and below.

The functions $F^{1}(t, x), \quad F_{k, s}^{1}(t, x), G^{1}(t, x), G_{k, s}^{1}(t, x), \quad F^{2}(t, x), \quad F_{k, s}^{2}(t, x), G^{2}(t, x)$, $G_{k, s}^{2}(t, x), F^{3}(t, x), G^{3}(t, x)$ where $k=\overline{1, r}, s=\overline{0, p_{1}}$, are known, sufficiently smooth and have all continuous derivatives occurring in the following relation and satisfying it:

$$
\begin{aligned}
& \sum_{j=0}^{p+2}\left(\left|\frac{\partial^{j}}{\partial x^{j}} F^{1}\right|+\left|\frac{\partial^{j}}{\partial x^{j}} G^{1}\right|+\left|\frac{\partial^{j}}{\partial x^{j}} F^{2}\right|+\left|\frac{\partial^{j}}{\partial x^{j}} G^{2}\right|+\left|\frac{\partial^{j}}{\partial x^{j}} F^{3}\right|+\left|\frac{\partial^{j}}{\partial x^{j}} G^{3}\right|+\right. \\
& \left.+\sum_{k=1}^{r} \sum_{s=0}^{p}\left(\left|\frac{\partial^{j}}{\partial x^{j}} F_{k, s}^{1}\right|+\left|\frac{\partial^{j}}{\partial x^{j}} G_{k, s}^{1}\right|+\left|\frac{\partial^{j}}{\partial x^{j}} F_{k, s}^{2}\right|+\left|\frac{\partial^{j}}{\partial x^{j}} G_{k, s}^{2}\right|\right)\right) \leqslant C, \quad \forall(t, x) \in G_{\left[0, t_{1}\right]} .
\end{aligned}
$$

Introduce the notation, where $k=0,1, \ldots, p+2$

$$
\begin{gathered}
\left\|h_{1}(t, x)\right\|_{1, p}=\sum_{j=0}^{p} \sup _{0<\xi \leqslant T} \sup _{x \in E_{1}}\left|\frac{\partial^{j}}{\partial x^{j}} h_{1}(\xi, x)\right|, h_{1}(t, x) \in Z_{x}^{p}\left(G_{[0, T]}\right), \\
\left\|h_{2}(x)\right\|_{2, p}=\sum_{j=0}^{p} \sup _{x \in E_{1}}\left|\frac{d^{j}}{d x^{j}} h_{2}(x)\right|, h_{2}(x) \in Z_{x}^{p}\left(G_{[0, T]}\right) . \\
\overline{U_{k}^{\tau}}(t)=\sup _{n \tau<\xi \leqslant t} \sup _{x \in E_{1}}\left|\frac{\partial^{k}}{\partial x^{k}} U^{\tau}(\xi, x)\right|, \\
\overline{V_{k}^{\tau}}(t)=\sup _{n \tau<\xi \leqslant t} \sup _{x \in E_{1}}\left|\frac{\partial^{k}}{\partial x^{k}} V^{\tau}(\xi, x)\right|, \quad n \tau<t \leqslant(n+1) \tau, \\
\overline{U_{k}}(0)=\sup _{x \in E_{1}}\left|\frac{\partial^{k}}{\partial x^{k}} U_{0}(x)\right|, \overline{V_{k}}(0)=\sup _{x \in E_{1}}\left|\frac{\partial^{k}}{\partial x^{k}} V_{0}(x)\right|, \\
\overline{U^{\tau}}(t)=\sum_{k=0}^{p+2} \overline{U_{k}^{\tau}}(t), \quad \overline{V^{\tau}}(t)=\sum_{k=0}^{p+2} \overline{V_{k}^{\tau}}(t), \bar{U}(0)=\sum_{k=0}^{p+2} \overline{U_{k}}(0), \quad \bar{V}(0)=\sum_{k=0}^{p+2} \overline{V_{k}}(0) .
\end{gathered}
$$


We use the method of weak approximation. The system of equations (15) is split into three fractional steps on differential level and time shift by $\frac{\tau}{3}$ in the traces of unknown functions

$$
\begin{gathered}
U_{t}^{\tau}(t, x)=3\left(a_{1}(t) U_{x x}^{\tau}(t, x)+b_{1}(t) U_{x}^{\tau}(t, x)\right), \quad n \tau<t \leqslant\left(n+\frac{1}{3}\right) \tau, \\
V_{t}^{\tau}(t, x)=3\left(a_{2}(t) V_{x x}^{\tau}(t, x)+b_{2}(t) V_{x}^{\tau}(t, x)\right), \\
U_{t}^{\tau}(t, x)=3 U^{\tau}\left(t-\frac{\tau}{3}, x\right) F^{1}+3 V^{\tau}\left(t-\frac{\tau}{3}, x\right) G^{1}, \\
V_{t}^{\tau}(t, x)=3 U^{\tau}\left(t-\frac{\tau}{3}, x\right) F^{2}+3 V^{\tau}\left(t-\frac{\tau}{3}, x\right) G^{2}, \\
U_{t}^{\tau}(t, x)=3 \sum_{k=1}^{r} \sum_{s=0}^{p_{1}}\left(\frac{\partial^{s}}{\partial x^{s}} U\left(t-\frac{\tau}{3}, a_{k}\right) F_{k, s}^{1}+\frac{\partial^{s}}{\partial x^{s}} V\left(t-\frac{\tau}{3}, a_{k}\right) G_{k, s}^{1}\right)+3 F^{3}, \\
V_{t}^{\tau}(t, x)=3 \sum_{k=1}^{r} \sum_{s=0}^{p_{1}}\left(\frac{\partial^{s}}{\partial x^{s}} U\left(t-\frac{\tau}{3}, a_{k}\right) F_{k, s}^{2}+\frac{\partial^{s}}{\partial x^{s}} V\left(t-\frac{\tau}{3}, a_{k}\right) G_{k, s}^{2}\right)+3 G^{3}, \\
\left(n+\frac{2}{3}\right) \tau<t \leqslant(n+1) \tau .
\end{gathered}
$$

On the first fractional step $t \in\left(0, \frac{\tau}{3}\right]$ we use the maximum principle, with the notation $(21)$. We obtain the following estimates

$$
\overline{U^{\tau}}(t) \leqslant \bar{U}(0), \quad \overline{V^{\tau}}(t) \leqslant \bar{V}(0)
$$

At the second and third fractional steps, differentiating with respect to $x$ from 0 up to order $p+2$ including, then integrating over the time variable and using the time shift in unknown functions, taking into account the notation (20), we obtain:

- on the second fractional step $t \in\left(\frac{\tau}{3}, \frac{2 \tau}{3}\right]$

$$
\begin{aligned}
& \overline{U^{\tau}}(t) \leqslant \overline{U^{\tau}}\left(\frac{\tau}{3}\right)+C \overline{U^{\tau}}\left(\frac{\tau}{3}\right) \tau+C \overline{V^{\tau}}\left(\frac{\tau}{3}\right) \tau, \\
& \overline{V^{\tau}}(t) \leqslant \overline{V^{\tau}}\left(\frac{\tau}{3}\right)+C \overline{U^{\tau}}\left(\frac{\tau}{3}\right) \tau+C \overline{V^{\tau}}\left(\frac{\tau}{3}\right) \tau,
\end{aligned}
$$

- on the third fractional step $t \in\left(\frac{2 \tau}{3}, \tau\right]$

$$
\begin{aligned}
& \overline{U^{\tau}}(t) \leqslant \overline{U^{\tau}}\left(\frac{2 \tau}{3}\right)+C\left(\overline{U^{\tau}}\left(\frac{2 \tau}{3}\right)+\overline{V^{\tau}}\left(\frac{2 \tau}{3}\right)+\sum_{k=0}^{p+2} \sup _{x \in E_{1} 0<\xi \leqslant T} \sup _{0<\xi}\left|\frac{\partial^{k}}{\partial x^{k}} F^{3}\right|\right) \tau, \\
& \overline{V^{\tau}}(t) \leqslant \overline{V^{\tau}}\left(\frac{2 \tau}{3}\right)+C\left(\overline{U^{\tau}}\left(\frac{2 \tau}{3}\right)+\overline{V^{\tau}}\left(\frac{2 \tau}{3}\right)+\sum_{k=0}^{p+2} \sup _{x \in E_{1} 0<\xi \leqslant T} \sup _{0<T}\left|\frac{\partial^{k}}{\partial x^{k}} G^{3}\right|\right) \tau .
\end{aligned}
$$

Considering inequalities (25), (26) and (27) with the notation (20) on the zero whole step we get

$$
U^{\tau}(t)+V^{\tau}(t) \leqslant e^{C \tau}\left(\bar{U}(0)+\bar{V}(0)+\sum_{k=0}^{p+2}\left(\sup _{x \in E_{1} 0<\xi \leqslant T} \sup _{0<}\left|\frac{\partial^{k}}{\partial x^{k}} F^{3}\right|+\sup _{x \in E_{1} 0<\xi \leqslant T} \sup _{0<\xi}\left|\frac{\partial^{k}}{\partial x^{k}} G^{3}\right|\right)\right)-
$$




$$
-\sum_{k=0}^{p+2}\left(\sup _{x \in E_{1} 0<\xi \leqslant T} \sup _{0<\xi}\left|\frac{\partial^{k}}{\partial x^{k}} F^{3}\right|+\sup _{x \in E_{1} 0<\xi \leqslant T} \sup _{\mid}\left|\frac{\partial^{k}}{\partial x^{k}} G^{3}\right|\right)
$$

On the interval $[0, T]$

$$
\begin{aligned}
& U^{\tau}(t)+V^{\tau}(t) \leqslant e^{C T}\left(\bar{U}(0)+\bar{V}(0)+\sum_{k=0}^{p+2}\left(\sup _{x \in E_{1} 0<\xi \leqslant T} \sup _{0<T}\left|\frac{\partial^{k}}{\partial x^{k}} F^{3}\right|+\right.\right. \\
&\left.\left.+\sup _{x \in E_{1}} \sup _{0<\xi \leqslant T}\left|\frac{\partial^{k}}{\partial x^{k}} G^{3}\right|\right)\right)-\sum_{k=0}^{p+2}\left(\sup _{x \in E_{1} 0<\xi \leqslant T} \sup _{k}\left|\frac{\partial^{k}}{\partial x^{k}} F^{3}\right|+\sup _{x \in E_{1} 0<\xi \leqslant T} \sup _{\mid}\left|\frac{\partial^{k}}{\partial x^{k}} G^{3}\right|\right) .
\end{aligned}
$$

These estimates guarantee the fulfilment of the conditions of the Arzela Compactness Theorem. By this theorem, some subsequences $U^{\tau_{k}}(t, x), V^{\tau_{k}}(t, x)$ of the sequences $U^{\tau}(t, x), V^{\tau}(t, x)$ of the solutions (22)-(24) of the split problem converge together with all relevant derivatives with respect to $x$ up to order $p$ to the functions $u(t, x), v(t, x)$ respectively, that according to the theorem of convergence of the weak approximation method is the solution to (1)-(2). Using this notation (19) we get

$$
\begin{aligned}
\|U(t, x)\|_{1, p}+\|V(t, x)\|_{1, p} \leqslant C\left(\left\|U_{0}(x)\right\|_{2, p+2}+\left\|V_{0}(x)\right\|_{2, p+2}\right. & \left.+\left\|F^{3}\right\|_{1, p+2}+\left\|G^{3}\right\|_{1, p+2}\right)- \\
& -\left(\left\|F^{3}\right\|_{1, p+2}+\left\|G^{3}\right\|_{1, p+2}\right) .
\end{aligned}
$$

Theorem (continuous dependence of the solution on the initial data). Assume that the condition (17) is satisfied, for $p \geqslant \max \left\{p_{1}, 2\right\} \geqslant 2$, so there exists at least one solution belonging to the class $Z_{x}^{p}\left(G_{[0, T]}\right)$ for which we have the estimate $\|U(t, x)\|_{1, p}+\|V(t, x)\|_{1, p} \leqslant$ $\leqslant C\left(\left\|U_{0}(x)\right\|_{2, p+2}+\left\|V_{0}(x)\right\|_{2, p+2}+\left\|F^{3}\right\|_{1, p+2}+\left\|G^{3}\right\|_{1, p+2}\right)-\left(\left\|F^{3}\right\|_{1, p+2}+\left\|G^{3}\right\|_{1, p+2}\right)$.

For the system of equations (1), (2) consider two sets of input data $u_{0}^{i}(x), v_{0}^{i}(x), f_{1}^{i}, f_{2}^{i} \in$ $Z_{x}^{p+4}\left(G_{[0, T]}\right)$, where $i=1,2, p \geqslant \max \left\{p_{1}, 2\right\}$. Then by the existence theorem the solution $u^{i}(t, x), v^{i}(t, x)$ exist in the class $Z_{x}^{p+2}\left(G_{[0, T]}\right)$. This solution satisfy the conditions of the uniqueness theorem for $p+2 \geqslant 4$. If $u^{i}(t, x), v^{i}(t, x) \in Z_{x}^{p+2}\left(G_{\left[0, t^{*}\right]}\right)$, then functions $f_{1}^{i}$ and $f_{2}^{i}$ satisfy the condition (3). We obtain

$$
\begin{gathered}
u_{t}^{i}(t, x)=a_{1}(t) u_{x x}^{i}(t, x)+b_{1}(t) u_{x}^{i}(t, x)+f_{1}^{i}\left(t, x, u^{i}, v^{i}, \bar{\varphi}_{u^{i}}(t), \bar{\varphi}_{v^{i}}(t)\right), \\
v_{t}^{i}(t, x)=a_{2}(t) v_{x x}^{i}(t, x)+b_{2}(t) v_{x}^{i}(t, x)+f_{2}^{i}\left(t, x, u^{i}, v^{i}, \bar{\varphi}_{u^{i}}(t), \bar{\varphi}_{v^{i}}(t)\right), \\
u^{i}(0, x)=u_{0}^{i}(x), \quad v^{i}(0, x)=v_{0}^{i}(x) .
\end{gathered}
$$

Introduce the notation $u(t, x)=u^{1}(t, x)-u^{2}(t, x), v(t, x)=v^{1}(t, x)-v^{2}(t, x), u_{0}(t, x)=$ $=u_{0}^{1}(t, x)-u_{0}^{2}(t, x), v_{0}(t, x)=v_{0}^{1}(t, x)-v_{0}^{2}(t, x)$. Subtracting the second system of equations from the first one we get the following

$$
\begin{gathered}
u_{t}(t, x)=a_{1}(t) u_{x x}(t, x)+b_{1}(t) u_{x}(t, x)+f_{1}^{1}\left(t, x, u^{1}, v^{1}, \bar{\varphi}_{u^{1}}(t), \bar{\varphi}_{v^{1}}(t)\right)- \\
-f_{1}^{1}\left(t, x, u^{2}, v^{2}, \bar{\varphi}_{u^{2}}(t), \bar{\varphi}_{v^{2}}(t)\right)+\overline{F^{3}}\left(t, x, u^{2}, v^{2}, \bar{\varphi}_{u^{2}}(t), \bar{\varphi}_{v^{2}}(t)\right), \\
v_{t}(t, x)=a_{2}(t) v_{x x}(t, x)+ \\
b_{2}(t) v_{x}(t, x)+f_{2}^{1}\left(t, x, u^{1}, v^{1}, \bar{\varphi}_{u^{1}}(t), \bar{\varphi}_{v^{1}}(t)\right)- \\
-f_{2}^{1}\left(t, x, u^{2}, v^{2}, \bar{\varphi}_{u^{2}}(t), \bar{\varphi}_{v^{2}}(t)\right)+\overline{G^{3}}\left(t, x, u^{2}, v^{2}, \bar{\varphi}_{u^{2}}(t), \bar{\varphi}_{v^{2}}(t)\right), \\
u(0, x)=u_{0}(x), v(0, x)=v_{0}(x),
\end{gathered}
$$

where 


$$
\begin{aligned}
& \overline{F^{3}}=f_{1}^{1}\left(t, x, u^{2}, v^{2}, \bar{\varphi}_{u^{2}}(t), \bar{\varphi}_{v^{2}}(t)\right)-f_{1}^{2}\left(t, x, u^{2}, v^{2}, \bar{\varphi}_{u^{2}}(t), \bar{\varphi}_{v^{2}}(t)\right), \\
& \overline{G^{3}}=f_{2}^{1}\left(t, x, u^{2}, v^{2}, \bar{\varphi}_{u^{2}}(t), \bar{\varphi}_{v^{2}}(t)\right)-f_{2}^{2}\left(t, x, u^{2}, v^{2}, \bar{\varphi}_{u^{2}}(t), \bar{\varphi}_{v^{2}}(t)\right) .
\end{aligned}
$$

By Conditions 1 and (18) we obtain

$$
\begin{gathered}
u_{t}(t, x)=a_{1}(t) u_{x x}(t, x)+b_{1}(t) u_{x}(t, x)+u(t, x) F^{1}+v(t, x) G^{1}+\sum_{k=1}^{r} \sum_{s=0}^{p_{1}}\left(\frac{\partial^{s}}{\partial x^{s}} u\left(t, a_{k}\right) F_{k, s}^{1}\right)+ \\
+\sum_{k=1}^{r} \sum_{s=0}^{p_{1}}\left(\frac{\partial^{s}}{\partial x^{s}} v\left(t, a_{k}\right) G_{k, s}^{1}\right)+\overline{F^{3}}\left(t, x, u^{2}, v^{2}, \bar{\varphi}_{u^{2}}(t), \bar{\varphi}_{v^{2}}(t)\right), \\
v_{t}(t, x)=a_{2}(t) v_{x x}(t, x)+b_{2}(t) v_{x}(t, x)+u(t, x) F^{2}+v(t, x) G^{2}+\sum_{k=1}^{r} \sum_{s=0}^{p_{1}}\left(\frac{\partial^{s}}{\partial x^{s}} u\left(t, a_{k}\right) F_{k, s}^{2}\right)+ \\
+\sum_{k=1}^{r} \sum_{s=0}^{p_{1}}\left(\frac{\partial^{s}}{\partial x^{s}} v\left(t, a_{k}\right) G_{k, s}^{2}\right)+\overline{G^{3}}\left(t, x, u^{2}, v^{2}, \bar{\varphi}_{u^{2}}(t), \bar{\varphi}_{v^{2}}(t)\right), \\
u(0, x)=u_{0}(x), \quad v(0, x)=v_{0}(x) .
\end{gathered}
$$

For the resulting problem using the Theorem (continuous dependence of the solution on the initial data), we get

$$
\begin{array}{r}
\|u(t, x)\|_{1, p}+\|v(t, x)\|_{1, p} \leqslant C\left(\left\|u_{0}(x)\right\|_{2, p+2}+\left\|v_{0}(x)\right\|_{2, p+2}+\left\|\overline{F^{3}}\right\|_{1, p+2}+\left\|\overline{G^{3}}\right\|_{1, p+2}\right)- \\
-\left(\left\|\overline{F^{3}}\right\|_{1, p+2}+\left\|\overline{G^{3}}\right\|_{1, p+2}\right) .
\end{array}
$$

Example. In the strip $\Pi_{[0, T]}=\left\{(t, x) \mid t \in[0, T], x \in E_{1}\right\}$ we consider the problem of finding real-valued functions $u(t, x), v(t, x), g(t)$ satisfying the system of equations

$$
\begin{array}{r}
u_{t}=u_{x x}+u v+b_{11}(t) u+b_{12}(t) v+m_{1}(t, x), \\
v_{t}=v_{x x}+b_{21}(t) u+b_{22}(t) v+g(t) m_{2}(t, x),
\end{array}
$$

the initial conditions

$$
u(0, x)=u_{0}(x), \quad v(0, x)=v_{0}(x), \quad x \in E_{1} .
$$

The solution satisfies the overdetermination condition

$$
v(t, 0)=\beta(t), \quad t \in[0, T],
$$

where $b_{i j}(t), m_{i}(t, x), u_{0}(x), v_{0}(x), \beta(t), i, j=1,2$ are given real-valued and bounded functions with their derivatives in $\Pi_{[0, T]}$. Let the all consistency conditions be fulfilled. Assume that the input data are sufficiently smooth and there is a solution in the class $Z_{x}^{6}\left(G_{[0, T]}\right)$. This is not difficult to prove, by analogy with reasoning in the article [1].

For the system of equations (28), (29) consider two sets of source functions $m_{1}^{i}(t, x), m_{2}^{i}(t, x)$, $i=1,2$, satisfying the conditions of the theorem of existence of the solution [1]. Then according to the theorem there exists a solution $u^{i}(t, x), v^{i}(t, x), g^{i}(t)$ for each set of input data. Let the following condition hold

$$
\left|m_{i}(t, 0)\right| \geqslant \delta>0, \quad i=1,2, \quad t \in[0, T], \quad \delta \text { - const. }
$$

Introduce the notation $U(t, x)=u^{1}(t, x)-u^{2}(t, x), V(t, x)=v^{1}(t, x)-v^{2}(t, x), Q(t)=$ $=g^{1}(t)-g^{2}(t)$. Subtracting the second system of equations from the first one we get

$$
\begin{gathered}
U_{t}=U_{x x}+u^{1} V+v^{2} U+b_{11}(t) U+b_{12}(t) V+m_{1}^{1}(t, x)-m_{1}^{2}(t, x), \\
V_{t}=V_{x x}+b_{21}(t) U+b_{22}(t) V+g^{1}(t)\left(m_{2}^{1}(t, x)-m_{2}^{2}(t, x)\right)+Q(t) m_{2}^{2}(t, x), \\
U(0, x)=0, \quad V(0, x)=0, \quad V(t, 0)=0 .
\end{gathered}
$$


Having reduced it to the auxiliary direct problem, we get the following

$$
\begin{gathered}
Q(t)=\frac{-V_{x x}(t, 0)-b_{21}(t) U(t, 0)-g^{1}(t)\left(m_{2}^{1}(t, 0)-m_{2}^{2}(t, 0)\right)}{m_{2}^{2}(t, 0)} \\
U_{t}=U_{x x}+u^{1} V+v^{2} U+b_{11}(t) U+b_{12}(t) V+m_{1}^{1}(t, x)-m_{1}^{2}(t, x) \\
V_{t}=V_{x x}+b_{21}(t) U+b_{22}(t) V+g^{1}(t)\left(m_{2}^{1}(t, x)-m_{2}^{2}(t, x)\right)+ \\
+m_{2}^{2}(t, x) \frac{-V_{x x}(t, 0)-b_{21}(t) U(t, 0)-g^{1}(t)\left(m_{2}^{1}(t, 0)-m_{2}^{2}(t, 0)\right)}{m_{2}^{2}(t, 0)} \\
U(0, x)=0, \quad V(0, x)=0 .
\end{gathered}
$$

The system (30), (31) is a special case of the system (15), (16).

$$
\begin{aligned}
& U_{t}=U_{x x}+\left(b_{11}(t)+v^{2}\right) U+\left(b_{12}(t)+u^{1}\right) V+m_{1}^{1}(t, x)-m_{1}^{2}(t, x) \\
& V_{t}=V_{x x}+b_{21}(t) U+b_{22}(t) V-\frac{m_{2}^{2}(t, x)}{m_{2}^{2}(t, 0)} V_{x x}(t, 0)-\frac{b_{21}(t) m_{2}^{2}(t, x)}{m_{2}^{2}(t, 0)} U(t, 0)+ \\
& \quad+g^{1}(t)\left(m_{2}^{1}(t, x)-m_{2}^{2}(t, x)\right)-\frac{g^{1}(t)\left(m_{2}^{1}(t, 0)-m_{2}^{2}(t, 0)\right) m_{2}^{2}(t, x)}{m_{2}^{2}(t, 0)} .
\end{aligned}
$$

Functions

$$
\begin{gathered}
F^{1}=b_{11}(t)+v^{2}, \quad F^{2}=b_{21}, \quad F_{1,2}^{1}=0, \quad F_{1,0}^{2}=-\frac{b_{21}(t) m_{2}^{2}(t, x)}{m_{2}^{2}(t, 0)}, \quad F^{3}=m_{1}^{1}(t, x)-m_{1}^{2}(t, x), \\
G^{1}=b_{12}(t)+u^{1}, \quad G^{2}=b_{22}(t), \quad G_{1,0}^{1}=0, \quad G_{1,2}^{2}=-\frac{m_{2}^{2}(t, x)}{m_{2}^{2}(t, 0)} \\
G^{3}=g^{1}(t)\left(m_{2}^{1}(t, x)-m_{2}^{2}(t, x)\right)-\frac{g^{1}(t)\left(m_{2}^{1}(t, 0)-m_{2}^{2}(t, 0)\right) m_{2}^{2}(t, x)}{m_{2}^{2}(t, 0)}
\end{gathered}
$$

satisfy Conditions 1 and (18), are known, sufficiently smooth and bounded functions.

Given the functions $u^{i}(t, x), v^{i}(t, x), g(t), i=1,2$ are classical solutions of the original problem, so they are continuous and bounded together with their derivatives in $Z_{x}^{4}\left(G_{[0, T]}\right)$. Using the theorem (continuous dependence of the solution on the initial data) taking into account the notation (19) we get

$$
\begin{aligned}
& \left\|F^{3}\right\| \leqslant C \sup _{0<\xi \leqslant T}\left|m_{1}^{1}(\xi, x)-m_{1}^{2}(\xi, x)\right| \leqslant C\left\|m_{1}^{1}(t, x)-m_{1}^{2}(t, x)\right\|_{1,6}, \\
& \left\|G^{3}\right\| \leqslant \sup _{x \in E_{1} 0<\xi \leqslant t} \sup _{0<}\left|g^{1}(t)\left(m_{2}^{1}(\xi, x)-m_{2}^{2}(\xi, x)\right)-\frac{g^{1}(\xi)\left(m_{2}^{1}(\xi, 0)-m_{2}^{2}(\xi, 0)\right) m_{2}^{2}(\xi, x)}{m_{2}^{2}(\xi, 0)}\right| \leqslant \\
& \leqslant C|| m_{2}^{1}(t, x)-m_{2}^{2}(t, x) \|_{1,6} \text {. } \\
& \|U(t, x)\|_{1,4}+\|V(t, x)\|_{1,4} \leqslant C\left(\left\|m_{1}^{1}(t, x)-m_{1}^{2}(t, x)\right\|_{1,6}+\left\|m_{2}^{1}(t, x)-m_{2}^{2}(t, x)\right\|_{1,6}\right)- \\
& \text { - }\left\|m_{1}^{1}(t, x)-m_{1}^{2}(t, x)\right\|_{1,6}+\left\|m_{2}^{1}(t, x)-m_{2}^{2}(t, x)\right\|_{1,6} . \\
& |Q| \leqslant \sup _{x \in E_{1}} \sup _{0<\xi \leqslant t}\left|\frac{-V_{x x}(\xi, 0)-b_{21}(\xi) U(\xi, 0)-g^{1}(\xi)\left(m_{2}^{1}(\xi, 0)-m_{2}^{2}(\xi, 0)\right)}{m_{2}^{2}(\xi, 0)}\right| \leqslant \\
& \leqslant C\left(\left\|m_{2}^{1}(t, x)-m_{2}^{2}(t, x)\right\|_{1,6}\right)
\end{aligned}
$$


Thus we have: if the input data are close, the corresponding solutions in the introduced norm are close too.

\title{
References
}

[1] O.A.Bender, T.N.Shipina, On the inverse Cauchy problem for a nonlinear parabolic system of equations, Proceedings of the XLII regional scientific student conference on mathematics and computer science, Krasnoyarsk, SFU, 2009, 52-54 (in Russian).

[2] I.V.Frolenkov, Yu.Ya.Belov, On the existence of solutions for a class loaded two-dimensional parabolic equations with the Cauchy data, non-classical equations of mathematical physics, Novosibirsk, Institut of Mathematics, 2012, 262-279 (in Russian).

[3] I.V.Frolenkov, M.A.Darzhaa, On the existence of solution of some problems for nonlinear loaded parabolic equations with Cauchy data, Journal of Siberian Federal University. Mathematics \& Physics, 7(2014), no. 2, 173-185.

[4] I.V.Frolenkov, G.V.Romanenko, On solvability of some special systems of one-dimensional loaded parabolic equations and composite-type systems with Cauchy data, Journal of Applied and Industrial Mathematics , 8(2014), no. 2, 196-207 (in Russian).

[5] G.A.Spichak, T.N.Shipina, Problem of identification of coefficients in a non-linear system of equations of parabolic type, Intern. conf. dedicated to the 80 birth anniversary of acad. M.M.Lavrentyev "Inverse and incorrect problems of mathematical physics", Novosibirsk, Sib. scien. publish., 2012.

[6] M.A.Yarovaya, I.V.Frolenkov, On the Cauchy problem for a one-dimensional loaded parabolic equation of special form, Graduate work, Siberian Federal University, Krasnoyarsk, 2015 (in Russian).

\section{О единственности и непрерывной зависимости от входных данных решения системы двух нагруженных параболических уравнений с данными Коши}

\author{
Игорь В .Фроленков \\ Ирина С. Антипина \\ Наталья М. Терских \\ Институт математики и фундаментальной информатики \\ Сибирский федеральный университет \\ Свободный, 79, Красноярск, 660041 \\ Россия
}

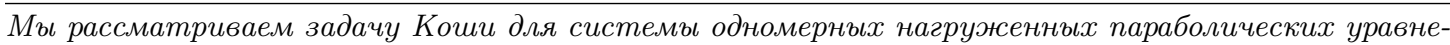
ний. Доказана единственность и непрерывная зависимость решений от входных данных в классе гладких ограниченных функций.

Ключевые слова: нагруженное уравнение, система параболических уравнений, метод слабой аппроксимачии, задача Коши, единственность, непрерывная зависимость решения от входных данHux. 